\title{
Spatiotemporal Control of CRISPR/Cas9 Function in Cells and Zebrafish using Light-Activated Guide RNA
}

Wenyuan Zhou ${ }^{1}$, Wes Brown ${ }^{1}$, Anirban Bardhan ${ }^{1}$, Michael Delaney ${ }^{2}$, Amber S. Ilk ${ }^{2}$, Randy R. Rauen ${ }^{2}$, Shoeb I. Kahn' ${ }^{2}$ Michael Tsang ${ }^{3}$, and Alexander Deiters ${ }^{1 *}$

${ }^{1}$ Department of Chemistry, University of Pittsburgh, Pittsburgh, Pennsylvania 15260, deiters@pitt.edu; ${ }^{2}$ Horizon Discovery, 2650 Crescent Drive, Lafayette, Colorado 80026; ${ }^{3}$ Department of Developmental Biology, School of Medicine, University of Pittsburgh, Pittsburgh, Pennsylvania 15260

\begin{abstract}
We developed a new method for conditional regulation of CRISPR/Cas9 activity in mammalian cells and zebrafish embryos via photochemically activated, caged guide RNAs. Caged gRNAs are generated by substituting four nucleobases evenly distributed throughout the 5 '-protospacer region with caged nucleobases during synthesis. Caging confers complete suppression of gRNA:target dsDNA hybridization and rapid restoration of CRISPR/Cas9 function upon optical activation. This tool offers simplicity and complete programmability in design, high spatiotemporal specificity in cells and zebrafish embryos, excellent off to on switching, and stability by preserving the ability to form Cas9:gRNA ribonucleoprotein complexes. caged gRNAs are novel tools for conditional control of gene editing thereby enabling the investigation of spatiotemporally complex physiological events by obtaining a better understanding of dynamic gene regulation.
\end{abstract}

\section{Introduction}

Adapted from the prokaryotic acquired immune system, CRISPR/Cas9 has been extensively studied and meticulously developed for its advantage in efficient and precise genome editing in a customizable fashion. ${ }^{1-5}$ As an RNA-guided DNA endonuclease, Cas9 protein first binds to a guide RNA (gRNA), which then enables site recognition by Cas9 on the target locus through Watson-Crick base pairing between the 5'20 nucleotide protospacer region of the gRNA and the desired DNA sequence. Subsequent cleavage of the target locus is then carried out by the nuclease domains, $\mathrm{HNH}$ and RuvC, of Cas9. ${ }^{6}$ Recent developments of the CRISPR/Cas9 system includes broad genomic targetability enabled by Cas 9 variants with PAM promiscuity, ${ }^{7}$ gene activation and repression, ${ }^{8-9}$ nucleobase editing, ${ }^{10}$ genomic loci imaging, ${ }^{11}$ and epigenetic modifications. ${ }^{12}$ Based on these developments, growing concerns of unwanted genomic manipulation ${ }^{13}$ and desire for synchronization of CRISPR/Cas 9 activity with precisely orchestrated genetic networks need to be addressed. 
Aiming at higher genomic editing precision by limiting the window of CRISPR/Cas9 activity as well as probing of spatiotemporally controlled gene function, researchers have endeavored to broaden the CRISPR/Cas9 toolkit for conditional control of its activity. ${ }^{14-16}$ Such efforts include small moleculeinduced Cas9 protein activation ${ }^{17-20}$ or reassembly, ${ }^{21}$ light activation of caged Cas9, ${ }^{22}$ reconstitution of single-chain Cas $9^{23}$ and split-Cas $9,{ }^{24}$ as well as optically controlled recruitment of transcription factors to catalytically dead Cas9 (dCas9) ${ }^{25-26}$ Amongst these developments, much effort was put into the regulation of the Cas 9 protein to restore its function upon external stimulation. These methods inevitably require the painstaking steps of protein engineering, including the screening of mutations and split sites, ${ }^{21,}{ }^{24}$ directed evolution, ${ }^{17}$ or unnatural amino acid mutagenesis. ${ }^{22}$ We anticipate that conditional control of chemically modified gRNA will not only circumvent the need for protein engineering, but will also provide a more easy-to-design and direct path to regulating the interaction between Cas9:gRNA ribonucleoprotein (RNP) complex and the target dsDNA. Several previous reports have shed light on this path, including using cleavable antisense-DNA as a protector for gRNA activity, ${ }^{27}$ ligand-dependent RNA cleavage and deprotection, ${ }^{28}$ ligand-dependent recruitment of transcriptional activators to dCas $9,{ }^{29}$ and small molecule-induced reassembly of the Cas9:gRNA complex. ${ }^{30}$ These designs, however, still are limited by the requirement for a third cellular component ${ }^{27-28}$ or reduced gRNA stability due to inability of RNP complex formation before activation. ${ }^{30-32}$ This is particularly important, as RNP delivery has been established as a universal approach for gene editing in different tissues and species with high efficiency and specificity, compared to alternative editing modalities. ${ }^{33-36}$

We henceforth introduce a photocaged gRNA design for the direct regulation of the interaction between RNP and dsDNA using light and demonstrate its application in an animal model. 6-Nitropiperonyloxymethyl (NPOM)-caged nucleobases have been successfully applied in the light-triggering of nucleic acid base-pairing in many living organisms. ${ }^{37-40}$ We employed NPOM-caged uridine and guanosine (Figure 1a) for application of this approach to a select set of target sequences in both mammalian cells and zebrafish embryos (Figure 1b). By replacing regular nucleobases with NPOM-caged nucleobases within the protospacer region of the gRNA, we anticipated that Cas9:gRNA:dsDNA ternary complex formation is inhibited until photolysis restores the base-pairing capability of the gRNA, while Cas9:caged gRNA interactions remain undisturbed (Figure 1c). This design is based on the rationale that the placement of NPOM-caging groups should ensure fast and complete photolysis to optically restore hybridization of an otherwise inaccessible protospacer of the gRNA. Our past experience has shown that very little background activity and excellent off $\rightarrow$ on switching upon light activation is achieved by installing one caging group every 5-6 nucleobases, evenly distributed throughout the oligonucleotide. ${ }^{41-43}$ We further envisioned that the Cas9:caged gRNA RNP can be pre-assembled and delivered as a complex for improved gRNA stability, ${ }^{32}$ facilitating application in both cultured mammalian cells as well as zebrafish embryos by lipid-mediated transfection ${ }^{44}$ and microinjection, ${ }^{23}$ 
respectively. Despite its synthetic challenge, we pursued a single caged gRNA because several studies have demonstrated better stability compared to the combination of crRNA (CRISPR RNA) and tracrRNA (transactivating crRNA), when complexed with Cas9 protein. ${ }^{45-46}$

\section{Results \& Discussion}

As a proof of concept, we first substituted four uridines evenly distributed within the 20 nt basepairing region of the gRNA with photocaged uridines for complete blockade of gRNA:dsDNA hybridization. ${ }^{47}$ To test if caged gRNA can fully suppress base pairing and restore gRNA:dsDNA hybridization upon irradiation with UV light, gel shift assays of Cas9:gRNA complex against 32Plabelled target dsDNA were conducted with noncaged gRNA (DsRed gRNA, nomenclature is used similarly for all other genes), no gRNA, and caged gRNA (DsRed-4U gRNA) in the presence or absence of 2 min irradiation (365 nm). The binding ability of caged gRNA to dsDNA was suppressed while light-induced decaging was shown to completely restore interaction of the gRNA with the complementary dsDNA (Figure 1d). Importantly, both non-caged and caged gRNAs bind to the Cas 9 protein with similar affinity, demonstrating that the caging of the protospacer region of gRNA does not interfere with formation of the Cas9:gRNA RNP complex (see Supporting Information).

Inspired by the successful optical control of the interaction between the RNP and the dsDNA, we designed photocaged gRNAs targeting different loci in both mammalian cells and zebrafish embryos a)

b)
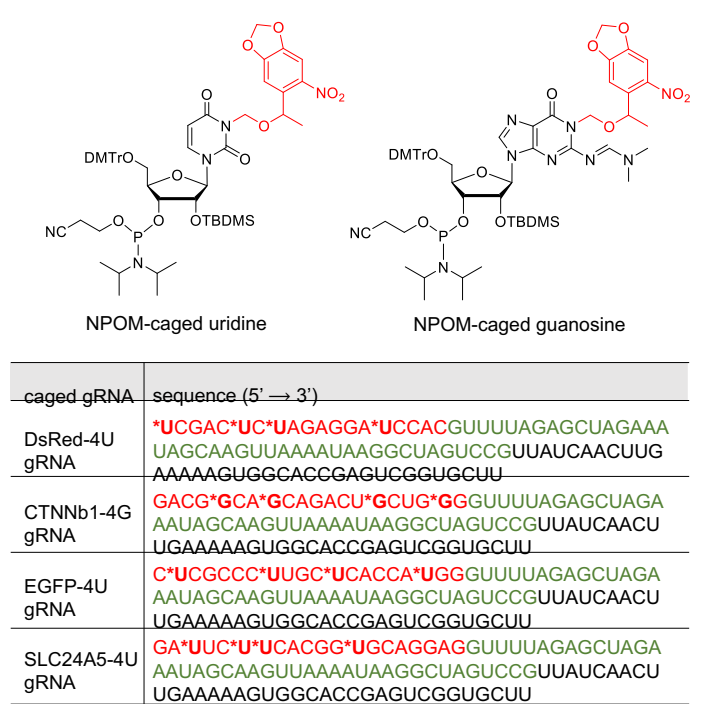

c)

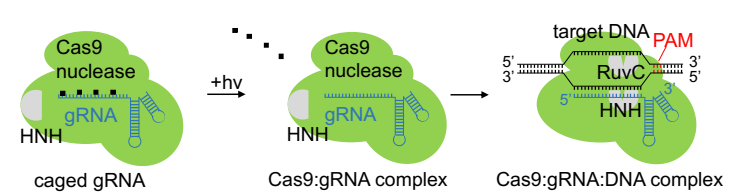

d)

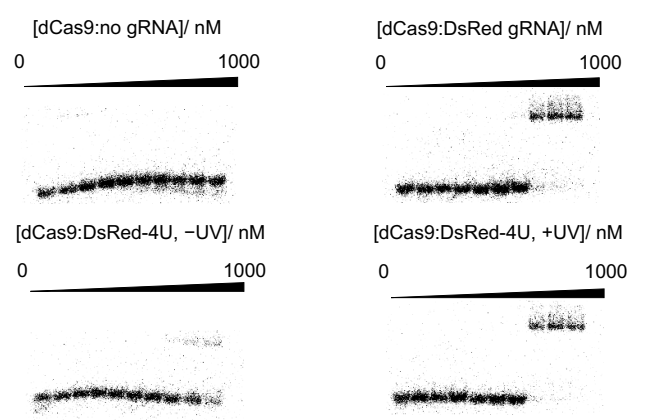

Figure 1. a) Structure of NPOM-protected uridine and guanosine with photocleavable caging groups shown in red. b) Sequences of photocaged gRNAs. The photocaged nucleotides are labelled by asterisks and the $20 \mathrm{nt}$ base-pairing region of the gRNA is shown in red. The Cas9 binding region is shown in green and the $S$. pyogenes terminator region is shown in black. The corresponding noncaged gRNAs (DsRed, CTNNb1, EGFP, and SLC24A5) have the exact same sequences without the nucleobase caging groups. c) The NPOMphotocaging groups are designed to abolish RNP binding to the target dsDNA until they are photochemically cleaved, thereby generating an active Cas9:gRNA complex. d) Phosphorautoradiography of gel shift assays demonstrates that the photocaged gRNA abolishes the binding affinity of Cas9 to target ${ }^{32} \mathrm{P}$-labelled dsDNA and that binding is fully restored upon light activation. following the developed strategy (Figure 1b). We first tested the optical triggering of CRISPR/Cas9 activity in mammalian cells transfected with a dual-fluorescence reporter plasmid. ${ }^{48}$ Targeted cleavage 
by Cas 9 endonuclease both at the beginning and at the end of the DsRed-polyA gene cassette results in cells switching from expressing DsRed to expressing EGFP (Figure 2a). HEK293T cells harboring the dual reporter plasmid were transfected with Cas9:EGFP gRNA together with Cas9:DsRed gRNA or Cas9:DsRed-4U gRNA RNPs, and were incubated for 6 hours before irradiation with $365 \mathrm{~nm}$ light. It should be noted that only one caged gRNA is needed in combination with EGFP gRNA to achieve full suppression of DsRed gene excision in the absence of optical triggering and efficient editing after illumination. The cells were then incubated for 72 hours, followed by imaging. EGFP expression was only observed in the case of light exposure, indicating activation of Cas 9 nuclease activity at the desired target sites by decaging of DsRed-4U gRNA while caged RNP-transfected cells that were kept in the dark remained inactive at the same minimal background level that is observed when no gRNA is present (Figure 2b). DsRed fluorescence is visible in all cells as DsRed fluorescent protein expressed before the activation of CRISPR/Cas 9 is highly stable and thus is also visualized at the time of imaging. Possible insufficient editing of the transiently transfected $\mathrm{pRG}$ reporter could also contribute to the observed DsRed fluorescence. Quantification of the fluorescent protein expression levels was carried out by using ImageJ software. Background was first subtracted based on a fixed value determined by the fluorescence intensity of non-transfected cells. ${ }^{49}$ Then the fluorescence intensity of each channel for all the cells in one well was integrated to represent the expression level of the fluorescent protein (Figure 2c). ${ }^{50}$

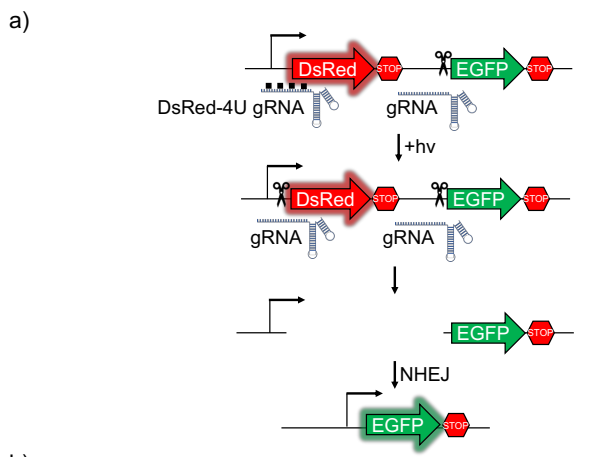

b)
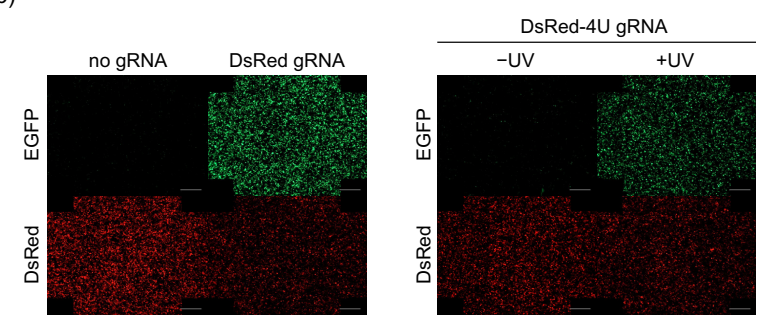

c)

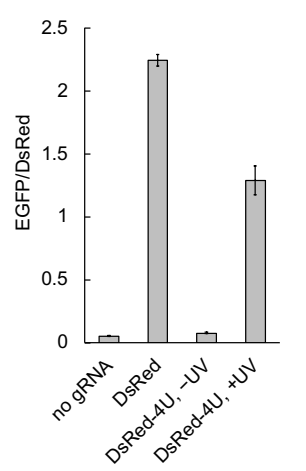

d)

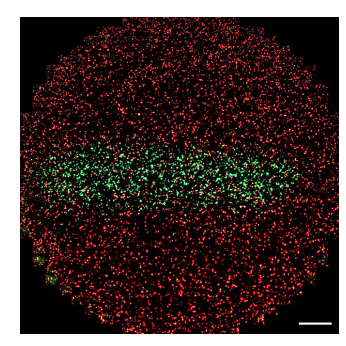

Figure 2. a) Schematic of the pRG reporter plasmid. Upon light activation, both functional gRNAs allow excision of the DsRed-terminator cassette from the pRG reporter plasmid and NHEJ repair leads to the expression of EGFP. b) HEK293T cells transfected with the pRG reporter plasmid, followed by delivery of Cas9:gRNA RNP complexes, were treated with or without $365 \mathrm{~nm}$ irradiation. EGFP expression was only observed with non-caged gRNA or when the caged gRNA was photochemically activated (scale bar $=100$ $\mu \mathrm{m})$. c) Quantification of EGFP and DsRed fluorescence was conducted by integration of fluorescence intensity in three independently transfected and treated wells for each condition using ImageJ software. d) Spatial control of lightactivated Cas9:gRNA function through patterned irradiation. HEK293T cells expressing the pRG reporter plasmid and transfected with Cas9:caged gRNA complex were exposed to $365 \mathrm{~nm}$ irradiation through a $2 \mathrm{~mm}$-wide slit in an mask (scale bar = $100 \mu \mathrm{m})$. 
Optical control of caged gRNA presents an opportunity for precise spatial activation. Indeed, only cells exposed to $365 \mathrm{~nm}$ light through a slitcontaining mask produced EGFP fluorescence, while all non-exposed cells only displayed DsRed expression (Figure 2d).

To demonstrate applicability of the developed optical tool to editing of the mammalian genome, we used a reported gRNA sequence (Figure $1 \mathrm{~b}$ ) to target a mammalian genomic locus within the CTNNb1 gene. ${ }^{51}$ Here, NPOM-caged guanosine (Figure 1a) was used instead of NPOM-caged uridine in order to achieve an even distribution of caged nucleobases following the methodology. Either CTNNb1 gRNA or caged CTNNb1-4G gRNA were delivered to HEK293T cells as Cas9 RNP complexes. Light activation was carried through exposure to $365 \mathrm{~nm}$ light 6 hours after delivery and gene editing was allowed for 3 days before cells were collected, followed cell lysis, and amplification of the genomic target site by nested PCR. Sanger sequencing of the amplicon and TIDE (Tracking of Indels by DEcomposition) analysis ${ }^{52}$ showed indel formation with $23.9 \%$ frequency for CTNNb1 gRNA a)

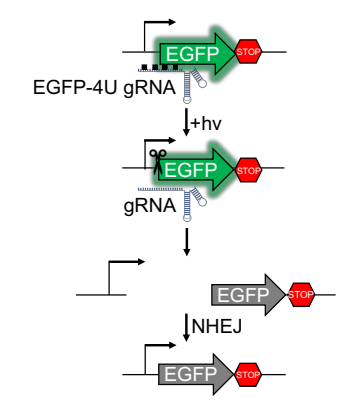

c)

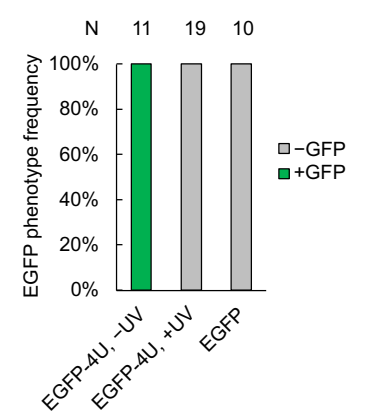

b)
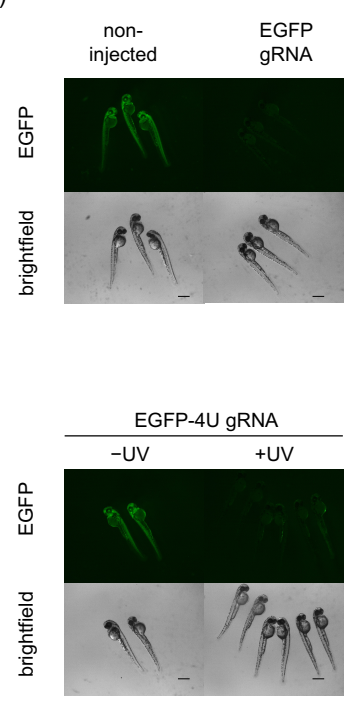

Figure 3. a) Schematic of the transgenic fish line fluorescent reporter. The gRNA recognizes the start codon region of EGFP, mutates it upon editing, and thus abolishes expression of the fluorescent protein. b) Representative micrographs of zebrafish at $48 \mathrm{hpf}$. Non-injected embryos demonstrate strong EGFP expression, while Cas9 RNP-injected embryos show complete loss of EGFP expression. c) Editing ability is blocked in caged EGFP-4U gRNA until irradiation with $365 \mathrm{~nm}$ light at 1 hour post-injection, indicating optical control of gene editing in embryos. Phenotype frequencies of the injected embryos at $48 \mathrm{hpf}$ are shown. Scale bars $=300 \mu \mathrm{m}$.

and $32.2 \%$ frequency for light-activated CTNNb1-4G gRNA, while virtually no background editing was detected in the absence of irradiation of CTNNb1-4G gRNA (0.7\% frequency). These results demonstrate that we are efficiently editing the mammalian cell genome with light-activated CTNNb1$4 G$ gRNA to a similar extend as the non-caged gRNA, while no editing was observed in the absence of irradiation - showcasing the excellent off $\rightarrow$ on switching of our caged gRNA methodology.

The Cas9:gRNA RNP complex is an excellent tool for gene editing in aquatic embryos, due to ease of assembly and injection into the fertilized oocyte. ${ }^{23}$ Furthermore, optical control is a powerful approach for conditional gene editing in zebrafish, because the embryos are transparent during the most important stages of development, allowing for irradiation of all tissues. To demonstrate the utility of photocaged gRNAs to control Cas9 gene editing in zebrafish, we first injected RNPs assembled with a caged gRNA targeting the start codon of EGFP (EGFP-4U) in the genome of a transgenic fish line (Tg(ubi:loxP-EGFP-loxP-mCherry) (Figure 3a). ${ }^{53}$ Disruption of the start codon prevents EGFP expression, as demonstrated by representative micrographs after application of non-caged gRNA 
(Figure 3b). Optical activation of EGFP-4U RNP complexes in embryos had similar editing efficiency as the non-caged gRNA containing RNP, significantly reducing EGFP expression in all embryos, as determined by fluorescent imaging at 48 hours post-fertilization (hpf) (Figure 3c) and fluorescence intensity measurements (see Supporting Information). Some mosaicism can be seen at levels similar to mosaicism from RNP injection previously reported. No toxicity was observed from exposure to $365 \mathrm{~nm}$ light (Supporting Information).

In order to further validate the universal applicability of this methodology, we next targeted the slc $24 a 5$ gene, ${ }^{54}$ an endogenous gene in zebrafish which is important for development of pigmentation by $48 \mathrm{hpf}$. s/c24a5 has been edited with Cas9 RNP injection before ${ }^{55-56}$ and the lack of pigmentation induced

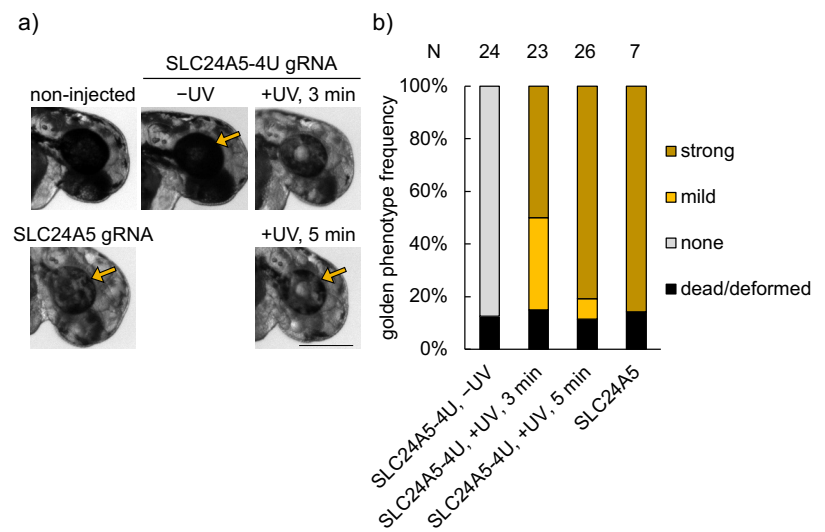

Figure 4. a) Representative images of embryos injected with RNPs assembled from non-caged SLC24A5 gRNA or caged SLC24A5-4U gRNA, then non-irradiated or irradiated $(365 \mathrm{~nm})$ for 3 or $5 \mathrm{~min}$. Images were recorded at $48 \mathrm{hpf}$. Arrows point to the retina, demonstrating loss of pigmentation through successful editing of the SLC24A5 locus. Scale bar = $300 \mu \mathrm{m}$. b) Phenotype frequencies of the injected embryos at $48 \mathrm{hpf}$. The golden phenotype was measured based on retinal pigmentation, with mild representing small patches of pigment loss, and strong representing a majority or complete retinal pigment loss. No background activity was observed for SLC24A5-4U, while $365 \mathrm{~nm}$ exposure for $5 \mathrm{~min}$ activated gene editing to the same level as the noncaged gRNA.

by editing of this locus is commonly referred to as the golden phenotype, most robustly observed as pigment loss in the retina of the developing animal. We used the same gRNA sequence that has been previously used for disrupting s/c24a5 function. ${ }^{56}$ Four uridine bases in the protospacer region were replaced with NPOM-caged uridines and showed complete inhibition of Cas 9 editing function until it was restored upon irradiation with $365 \mathrm{~nm}$ light. Resulting gene editing led to almost complete loss of retinal pigmentation (Figure 4a). Increasing light exposure (5 min vs $3 \mathrm{~min}$ ) resulted in increased pigmentation loss in a larger proportion of embryos, demonstrating that this method allows for tuning of editing efficiency and that full optical activation of Cas9 RNP function can be achieved (Figure 4b, and Supporting Information). Optical off to on switching of gene editing is further confirmed by TIDE analysis of the s/c24a5 gene locus, showing a 75\% indel rate for non-caged SLC24A5 gRNA, 84\% for light-triggered SLC24A5-4U gRNA, and 7\% for SLC24A5-4U gRNA in the absence of irradiation.

\section{Summary}

We developed a new method to optically control CRISPR/Cas9 activity through nucleobase-caged gRNAs, thereby further expanding the light-activated tool set available for conditionally controlled gene editing with spatial and temporal resolution. ${ }^{57-59}$ We successfully applied this approach in both 
mammalian cells and zebrafish embryos with high efficiency on both transiently transfected plasmid DNA and genomic loci. NPOM-caged gRNAs expand the gene editing toolbox with unique features, including 1) rapid and non-invasive activation of CRISPR/Cas9 activity, 2) precise spatiotemporal control, 3) modularity and programmability of the light-controlled gRNA sequence, 4) formation of a stable Cas9:gRNA complex from commercially available protein, 5) broad applicability for delivery into cells and organisms in the form of RNP complexes, and 6) capability for tuning of gene editing efficiency. We expect that NPOM-caged gRNAs will find utility in the dissection of regulatory networks in the rapidly developing zebrafish embryo in a temporally and spatially sensitive manner. Furthermore, the light-activated Cas9:gRNA RNP system is expected to be functional in other cell lines and (aquatic) embryos.

\section{Acknowledgements}

This work was supported by the National Institutes of Health (R21HD085206 and R01GM112728 to $A D ; T 32 G M 088119$ to $W B)$.

\section{References}

1. Adli, M., The CRISPR tool kit for genome editing and beyond. Nat Commun 2018, 9 (1), 1911.

2. Wang, H.; La Russa, M.; Qi, L. S., CRISPR/Cas9 in Genome Editing and Beyond. Annu Rev Biochem 2016, 85, 227-64.

3. Wright, A. V.; Nunez, J. K.; Doudna, J. A., Biology and Applications of CRISPR Systems: Harnessing Nature's Toolbox for Genome Engineering. Cell 2016, 164 (1-2), 29-44.

4. Barrangou, R.; Doudna, J. A., Applications of CRISPR technologies in research and beyond. Nat Biotechnol 2016, 34 (9), 933-941.

5. Komor, A. C.; Badran, A. H.; Liu, D. R., CRISPR-Based Technologies for the Manipulation of Eukaryotic Genomes. Cell 2017, 168 (1-2), 20-36.

6. Jiang, F.; Doudna, J. A., CRISPR-Cas9 Structures and Mechanisms. Annu Rev Biophys 2017, 46, 505-529.

7. Hu, J. H.; Miller, S. M.; Geurts, M. H.; Tang, W.; Chen, L.; Sun, N.; Zeina, C. M.; Gao, X.; Rees, H. A.; Lin, Z.; Liu, D. R., Evolved Cas9 variants with broad PAM compatibility and high DNA specificity. Nature 2018, 556 (7699), 57-63.

8. Konermann, S.; Brigham, M. D.; Trevino, A. E.; Joung, J.; Abudayyeh, O. O.; Barcena, C.; Hsu, P. D.; Habib, N.; Gootenberg, J. S.; Nishimasu, H.; Nureki, O.; Zhang, F., Genome-scale transcriptional activation by an engineered CRISPR-Cas9 complex. Nature 2015, 517 (7536), 5838.

9. Gilbert, L. A.; Horlbeck, M. A.; Adamson, B.; Villalta, J. E.; Chen, Y.; Whitehead, E. H.; Guimaraes, C.; Panning, B.; Ploegh, H. L.; Bassik, M. C.; Qi, L. S.; Kampmann, M.; Weissman, J. S., GenomeScale CRISPR-Mediated Control of Gene Repression and Activation. Cell 2014, 159 (3), 647-61.

10. Eid, A.; Alshareef, S.; Mahfouz, M. M., CRISPR base editors: genome editing without doublestranded breaks. Biochem J 2018, 475 (11), 1955-1964. 
11. Chen, B.; Gilbert, L. A.; Cimini, B. A.; Schnitzbauer, J.; Zhang, W.; Li, G. W.; Park, J.; Blackburn, E. H.; Weissman, J. S.; Qi, L. S.; Huang, B., Dynamic imaging of genomic loci in living human cells by an optimized CRISPR/Cas system. Cell 2013, 155 (7), 1479-91.

12. Hilton, I. B.; D'Ippolito, A. M.; Vockley, C. M.; Thakore, P. I.; Crawford, G. E.; Reddy, T. E.; Gersbach, C. A., Epigenome editing by a CRISPR-Cas9-based acetyltransferase activates genes from promoters and enhancers. Nat Biotechnol 2015, 33 (5), 510-7.

13. Norris, A. L.; Lee, S. S.; Greenlees, K. J.; Tadesse, D. A.; Miller, M. F.; Lombardi, H., Template plasmid integration in germline genome-edited cattle. BioRxiv 2019, 715482.

14. Zhou, W.; Deiters, A., Conditional Control of CRISPR/Cas9 Function. Angew Chem Int Ed Engl 2016, 55 (18), 5394-9.

15. Nunez, J. K.; Harrington, L. B.; Doudna, J. A., Chemical and Biophysical Modulation of Cas 9 for Tunable Genome Engineering. ACS Chem Biol 2016, 11 (3), 681-8.

16. Gangopadhyay, S. A.; Cox, K. J.; Manna, D.; Lim, D.; Maji, B.; Zhou, Q.; Choudhary, A., Precision Control of CRISPR-Cas9 Using Small Molecules and Light. Biochemistry 2019, 58 (4), 234-244.

17. Davis, K. M.; Pattanayak, V.; Thompson, D. B.; Zuris, J. A.; Liu, D. R., Small molecule-triggered Cas9 protein with improved genome-editing specificity. Nat Chem Biol 2015, 11 (5), 316-8.

18. Liu, K. I.; Ramli, M. N.; Woo, C. W.; Wang, Y.; Zhao, T.; Zhang, X.; Yim, G. R.; Chong, B. Y.; Gowher, A.; Chua, M. Z.; Jung, J.; Lee, J. H.; Tan, M. H., A chemical-inducible CRISPR-Cas9 system for rapid control of genome editing. Nat Chem Biol 2016, 12 (11), 980-987.

19. Oakes, B. L.; Nadler, D. C.; Flamholz, A.; Fellmann, C.; Staahl, B. T.; Doudna, J. A.; Savage, D. F., Profiling of engineering hotspots identifies an allosteric CRISPR-Cas9 switch. Nat Biotechnol 2016, 34 (6), 646-51.

20. Rose, J. C.; Stephany, J. J.; Valente, W. J.; Trevillian, B. M.; Dang, H. V.; Bielas, J. H.; Maly, D. J.; Fowler, D. M., Rapidly inducible Cas9 and DSB-ddPCR to probe editing kinetics. Nat Methods 2017, 14 (9), 891-896.

21. Zetsche, B.; Volz, S. E.; Zhang, F., A split-Cas9 architecture for inducible genome editing and transcription modulation. Nat Biotechnol 2015, 33 (2), 139-42.

22. Hemphill, J.; Borchardt, E. K.; Brown, K.; Asokan, A.; Deiters, A., Optical Control of CRISPR/Cas9 Gene Editing. J Am Chem Soc 2015, 137 (17), 5642-5.

23. Xin, Y.; Duan, C., Microinjection of Antisense Morpholinos, CRISPR/Cas9 RNP, and RNA/DNA into Zebrafish Embryos. Methods Mol Biol 2018, 1742, 205-211.

24. Nihongaki, Y.; Kawano, F.; Nakajima, T.; Sato, M., Photoactivatable CRISPR-Cas9 for optogenetic genome editing. Nat Biotechnol 2015, 33 (7), 755-60.

25. Nihongaki, Y.; Yamamoto, S.; Kawano, F.; Suzuki, H.; Sato, M., CRISPR-Cas9-based photoactivatable transcription system. Chem Biol 2015, 22 (2), 169-74.

26. Polstein, L. R.; Gersbach, C. A., A light-inducible CRISPR-Cas9 system for control of endogenous gene activation. Nat Chem Biol 2015, 11 (3), 198-200.

27. Jain, P. K.; Ramanan, V.; Schepers, A. G.; Dalvie, N. S.; Panda, A.; Fleming, H. E.; Bhatia, S. N., Development of Light-Activated CRISPR Using Guide RNAs with Photocleavable Protectors. Angew Chem Int Ed Engl 2016, 55 (40), 12440-4.

28. Ferry, Q. R.; Lyutova, R.; Fulga, T. A., Rational design of inducible CRISPR guide RNAs for de novo assembly of transcriptional programs. Nat Commun 2017, 8, 14633.

29. Maji, B.; Moore, C. L.; Zetsche, B.; Volz, S. E.; Zhang, F.; Shoulders, M. D.; Choudhary, A., Multidimensional chemical control of CRISPR-Cas9. Nat Chem Biol 2017, 13 (1), 9-11.

30. Kundert, K.; Lucas, J. E.; Watters, K. E.; Fellmann, C.; Ng, A. H.; Heineike, B. M.; Fitzsimmons, C. M.; Oakes, B. L.; Savage, D. F.; El-Samad, H.; Doudna, J. A.; Kortemme, T., 2018. 
31. Jinek, M.; East, A.; Cheng, A.; Lin, S.; Ma, E.; Doudna, J., RNA-programmed genome editing in human cells. Elife 2013, 2, e00471.

32. Ma, H.; Tu, L. C.; Naseri, A.; Huisman, M.; Zhang, S.; Grunwald, D.; Pederson, T., CRISPR-Cas9 nuclear dynamics and target recognition in living cells. J Cell Biol 2016, 214 (5), 529-37.

33. DeWitt, M. A.; Corn, J. E.; Carroll, D., Genome editing via delivery of Cas9 ribonucleoprotein. Methods 2017, 121-122, 9-15.

34. Staahl, B. T.; Benekareddy, M.; Coulon-Bainier, C.; Banfal, A. A.; Floor, S. N.; Sabo, J. K.; Urnes, C.; Munares, G. A.; Ghosh, A.; Doudna, J. A., Efficient genome editing in the mouse brain by local delivery of engineered Cas9 ribonucleoprotein complexes. Nat Biotechnol 2017, 35 (5), 431-434.

35. Glass, Z.; Lee, M.; Li, Y.; Xu, Q., Engineering the Delivery System for CRISPR-Based Genome Editing. Trends Biotechnol 2018, 36 (2), 173-185.

36. Lin, S.; Staahl, B. T.; Alla, R. K.; Doudna, J. A., Enhanced homology-directed human genome engineering by controlled timing of CRISPR/Cas9 delivery. Elife 2014, 3, e04766.

37. Liu, Q.; Deiters, A., Optochemical control of deoxyoligonucleotide function via a nucleobasecaging approach. Acc Chem Res 2014, 47 (1), 45-55.

38. Hemphill, J.; Govan, J.; Uprety, R.; Tsang, M.; Deiters, A., Site-specific promoter caging enables optochemical gene activation in cells and animals. J Am Chem Soc 2014, 136 (19), 7152-8.

39. Govan, J. M.; Young, D. D.; Lusic, H.; Liu, Q.; Lively, M. O.; Deiters, A., Optochemical control of RNA interference in mammalian cells. Nucleic Acids Res 2013, 41 (22), 10518-28.

40. Deiters, A.; Lusic, H., A New Photocaging Group for Aromatic N-Heterocycles. Synthesis 2006, 2006 (13), 2147-2150.

41. Lusic, H.; Young, D. D.; Lively, M. O.; Deiters, A., Photochemical DNA activation. Organic letters 2007, 9 (10), 1903-6.

42. Young, D. D.; Edwards, W. F.; Lusic, H.; Lively, M. O.; Deiters, A., Light-triggered polymerase chain reaction. Chem Commun (Camb) 2008, (4), 462-4.

43. Young, D. D.; Lusic, H.; Lively, M. O.; Yoder, J. A.; Deiters, A., Gene silencing in mammalian cells with light-activated antisense agents. Chembiochem 2008, 9 (18), 2937-40.

44. Yu, X.; Liang, X.; Xie, H.; Kumar, S.; Ravinder, N.; Potter, J.; du Jeu, X. d. M.; Chesnut, J. D., Improved delivery of Cas9 protein/gRNA complexes using lipofectamine CRISPRMAX. Biotechnology letters 2016, 38 (6), 919-929.

45. Hendel, A.; Bak, R. O.; Clark, J. T.; Kennedy, A. B.; Ryan, D. E.; Roy, S.; Steinfeld, I.; Lunstad, B. D.; Kaiser, R. J.; Wilkens, A. B.; Bacchetta, R.; Tsalenko, A.; Dellinger, D.; Bruhn, L.; Porteus, M. $\mathrm{H}$., Chemically modified guide RNAs enhance CRISPR-Cas genome editing in human primary cells. Nat Biotechnol 2015, 33 (9), 985-989.

46. Ryan, D. E.; Taussig, D.; Steinfeld, I.; Phadnis, S. M.; Lunstad, B. D.; Singh, M.; Vuong, X.; Okochi, K. D.; McCaffrey, R.; Olesiak, M.; Roy, S.; Yung, C. W.; Curry, B.; Sampson, J. R.; Bruhn, L.; Dellinger, D. J., Improving CRISPR-Cas specificity with chemical modifications in single-guide RNAs. Nucleic Acids Res 2018, 46 (2), 792-803.

47. Connelly, C. M.; Uprety, R.; Hemphill, J.; Deiters, A., Spatiotemporal control of microRNA function using light-activated antagomirs. Mol Biosyst 2012, 8 (11), 2987-93.

48. De Gasperi, R.; Rocher, A. B.; Sosa, M. A.; Wearne, S. L.; Perez, G. M.; Friedrich, V. L., Jr.; Hof, P. R.; Elder, G. A., The IRG mouse: a two-color fluorescent reporter for assessing Cre-mediated recombination and imaging complex cellular relationships in situ. Genesis 2008, 46 (6), 308-17.

49. Furia, L.; Pelicci, P. G.; Faretta, M., A computational platform for robotized fluorescence microscopy (I): high-content image-based cell-cycle analysis. Cytometry A 2013, 83 (4), 333-43. 
50. Higuchi-Sanabria, R.; Garcia, E. J.; Tomoiaga, D.; Munteanu, E. L.; Feinstein, P.; Pon, L. A., Characterization of Fluorescent Proteins for Three- and Four-Color Live-Cell Imaging in S. cerevisiae. PLoS One 2016, 11 (1), e0146120.

51. Sanson, K. R.; Hanna, R. E.; Hegde, M.; Donovan, K. F.; Strand, C.; Sullender, M. E.; Vaimberg, E. W.; Goodale, A.; Root, D. E.; Piccioni, F.; Doench, J. G., Optimized libraries for CRISPR-Cas9 genetic screens with multiple modalities. Nat Commun 2018, 9 (1), 5416.

52. Brinkman, E. K.; Chen, T.; Amendola, M.; van Steensel, B., Easy quantitative assessment of genome editing by sequence trace decomposition. Nucleic Acids Res 2014, 42 (22), e168.

53. Mosimann, C.; Kaufman, C. K.; Li, P.; Pugach, E. K.; Tamplin, O. J.; Zon, L. I., Ubiquitous transgene expression and Cre-based recombination driven by the ubiquitin promoter in zebrafish. Development 2011, 138 (1), 169-177.

54. Lamason, R. L.; Mohideen, M. A.; Mest, J. R.; Wong, A. C.; Norton, H. L.; Aros, M. C.; Jurynec, M. J.; Mao, X.; Humphreville, V. R.; Humbert, J. E.; Sinha, S.; Moore, J. L.; Jagadeeswaran, P.; Zhao, W.; Ning, G.; Makalowska, I.; McKeigue, P. M.; O'Donnell, D.; Kittles, R.; Parra, E. J.; Mangini, N. J.; Grunwald, D. J.; Shriver, M. D.; Canfield, V. A.; Cheng, K. C., SLC24A5, a putative cation exchanger, affects pigmentation in zebrafish and humans. Science 2005, 310 (5755), 1782-6.

55. Burger, A.; Lindsay, H.; Felker, A.; Hess, C.; Anders, C.; Chiavacci, E.; Zaugg, J.; Weber, L. M.; Catena, R.; Jinek, M.; Robinson, M. D.; Mosimann, C., Maximizing mutagenesis with solubilized CRISPR-Cas9 ribonucleoprotein complexes. Development 2016, 143 (11), 2025.

56. Jao, L.-E.; Wente, S. R.; Chen, W., Efficient multiplex biallelic zebrafish genome editing using a CRISPR nuclease system. Proc Natl Acad Sci U S A 2013, 110 (34), 13904-13909.

57. Ankenbruck, N.; Courtney, T.; Naro, Y.; Deiters, A., Optochemical Control of Biological Processes in Cells and Animals. Angewandte Chemie 2018, 57 (11), 2768-2798.

58. Kowalik, L.; Chen, J. K., Illuminating developmental biology through photochemistry. Nat Chem Biol 2017, 13 (6), 587-598.

59. O'Banion, C. P.; Lawrence, D. S., Optogenetics: A Primer for Chemists. Chembiochem 2018, 19 (12), 1201-1216. 
bioRxiv preprint doi: https://doi.org/10.1101/831974; this version posted November 13,2019 . The copyright holder for this preprint (which was not certified by peer review) is the author/funder, who has granted bioRxiv a license to display the preprint in perpetuity. It is made available under aCC-BY-NC-ND 4.0 International license. 